\title{
Artikel
}

Youmna Fouad/Heike Greschke*

\section{"Wie ist das denn in deinem Heimatland?" Kommunikative Muster invektiver Kulturvergleiche im Orientierungskurs}


Abstract: The German Orientation Course is considered as one of the most important measures of integration policy in Germany. It is a mandatory German language and cultural course dedicated to refugees and immigrants. It aims to provide knowledge about the German political system and certain 'cultural' German values. This article examines the Orientation Course as an intercultural encounter, as a place which is institutionally and politically framed and also as a hierarchically didactic arena where cultural mediation takes place. It illustrates also how invective communication happens through the establishment of certain communicative patterns which can degrade or disparage social groups. Based on participatory observation in the Orientation Course using the Genre Analysis, this article argues in which ways these communicative patterns can affect the social order and unite or shape groups.

Keywords: invektive Kulturvergleiche, kommunikative Muster, Gattungsanalyse, Integrationskurs, Teilhabe; Othering, Geflüchtete, Integration - invective cultural comparisons, communication patterns, genre analysis, Integration Course, othering, refugees, integration

*Prof. Dr. Heike Greschke, Technische Universität Dresden, Institut für Soziologie, Professur für Soziologischen Kulturenvergleich und Qualitative Sozialforschung, heike.greschke@tu-dresden.de

Youmna Fouad, M.A., Technische Universität Dresden, Sonderforschungsbereich 1285 "Invektivität. Konstellationen und Dynamiken der Herabsetzung", Teilprojekt R, youmna.fouad@tu-dresden.de

\section{Einleitung}

Mit dem Integrationskurs wurde im Jahr 2005 auf Basis des Zuwanderungsgesetzes ein neues deutsches integrationspolitisches Instrument geschaffen, das sich ausschließlich an Zugewanderte und Geflüchtete richtet und damit Integrationserwartungen und Desintegrationsbefürchtungen eng an Migrationsphänomene koppelt. Für die Durchführung und Kontrolle der Integrationskurse ist das Bundesamt für Migration und Flüchtlinge verantwortlich. Der Integrationskurs besteht aus einem Sprachkurs mit 600 bzw. 900 Unterrichtsstunden zu je 45 Minuten und einem Orientierungskurs mit 100 Stunden. Nach dem erfolgreichen Abschluss des Sprachkurses besuchen die Teilnehmenden den Orientierungskurs. Das ,Zertifikat Integrationskurs' können Teilnehmende nur dann erlangen, wenn sie neben dem Sprachtest ,Deutsch für Zuwanderer' (DTZ) auch den Test ,Leben in Deutschland' (LID) nach dem Orientierungskurs erfolgreich bestanden haben. ${ }^{1}$ Dieser erfolgreiche Abschluss soll den Teilnehmenden bescheinigen,

1 BAMF (2017) Curriculum, S. 10. dass sie über ausreichende Deutschkenntnisse und wichtige Grundkenntnisse über die deutsche Rechtsordnung sowie die deutsche Gesellschaft verfügen und somit den Weg der Einbürgerung für sie erleichtern.

Der Orientierungskurs führt Zugewanderte an die Grundprinzipien des deutschen Staates und der deutschen Gesellschaft heran und soll es den Teilnehmenden ermöglichen, darüber in einen Dialog miteinander zu treten. Die Teilnehmenden sollen befähigt werden, sich im Alltag zu orientieren und am gesellschaftlichen Leben teilzunehmen. ${ }^{2}$

Ergänzend formuliert das Bundesamt für Migration und Flüchtlinge (BAMF) im Curriculum für einen bundesweiten Orientierungskurs dessen Funktion und Ziele: Die Zugewanderten sind gehalten, "sich auf ein näheres Kennenlernen des deutschen Staates und der deutschen Gesellschaft einzulassen und in einen positiven Dialog einzutreten, der den Weg für das längerfristige Ziel der Integration in Deutschland ebnet."3

2 Vgl. BAMF (2017) Curriculum, S. 7f. 3 BAMF (2017) Curriculum, S. 7. 
Der Orientierungskurs ist in seiner Konzeption und Didaktik an die politische Bildung und den Fremdsprachunterricht in Deutschland angelehnt. Die Fremdsprachendidaktik unterscheidet je nach Erwerbsumgebung zwischen ,Deutsch als Fremdsprache' (DaF) und ,Deutsch als Zweitsprache' (DaZ). Der Orientierungskurs wird demnach dem DaZ-Bereich zugeordnet, weil Deutsch als Landessprache in Deutschland erlernt wird. Anders verhält es sich, wenn vor der Migration, etwa in einem Goethe-Institut, Deutsch gelernt wird. Diese Spracherwerbsform wird dem DaFBereich zugeordnet. ${ }^{4}$ Der für die vorliegende Untersuchung wesentliche Unterschied zwischen beiden Bereichen besteht darin, dass die Erwartungen an erwachsene Sprachlernende im DaFUnterricht weder institutionell noch politisch eingebettet sind, hingegen hat der Integrationskurs als Spracherwerbsform aus dem DaZ-Bereich in Deutschland eine deutliche politische und institutionelle Rahmung. Im DaF-Bereich entscheiden sich die erwachsenen Sprachlernenden i.d.R. frei, ob sie Deutsch als Fremdsprache lernen und Zertifikate erlangen wollen, ganz im Gegenteil zu den Sprachlernenden im Integrationskurs, insbesondere zu jenen zur Teilnahme Verpflichteten, für die das Ergebnis der anschließenden Prüfungen mit Konsequenzen für ihren Aufenthaltsstatus verbunden ist. ${ }^{5}$ Als weiteres Unterscheidungsmerkmal hebt Fornhoff die Bedeutung der Kulturvermittlung und des kulturbezogenen Lernens hervor.

Während im DaF-Bereich kulturbezogenes Deutungswissen und kulturbezogene Deutungskompetenzen nur im Sinne ihrer Kenntnis und davon ausgehend ihrer möglichen Nutzung in inter- und transkulturellen Kommunikations-und Austauschprozessen angeeignet werden müssen, ihr Erwerb also zunächst einmal zu nichts ver-

4 Vgl. Gehring (2018) Fremdsprache, S. 9.

5 „Kommt ein Ausländer seiner Teilnahmepflicht aus von ihm zu vertretenden Gründen nicht nach oder legt er den Abschlusstest nicht erfolgreich ab, weist ihn die zuständige Ausländerbehörde vor der Verlängerung seiner Aufenthaltserlaubnis auf die möglichen Auswirkungen seines Handelns ( $\S 8$ Abs. 3, § 9 Abs. 2 Satz 1 Nr. Vgl. 3 des Staatsangehörigkeitsgesetzes) hin. Die Ausländerbehörde kann den Ausländer mit Mitteln des Verwaltungszwangs zur Erfüllung seiner Teilnahmepflicht anhalten. Bei Verletzung der Teilnahmepflicht kann der voraussichtliche Kostenbeitrag auch vorab in einer Summe durch Gebührenbescheid erhoben werden." AufenthG § 44a. pflichtet, richtet sich das kulturbezogene Lernen im DaZ-Bereich insgesamt auf ein viel weitergehendes Ziel: nämlich auf die Integration der Lernenden in die deutsche Gesellschaft und damit auf einen Vorgang, der zwar auch wissensbasiert ist und Deutungskompetenzen verlangt, zugleich aber mit der Anerkennung von Werten und der Übernahme von Pflichten verbunden ist. ${ }^{6}$

Für das kulturbezogene Lernen wird in beiden Bereichen auf die kulturvergleichende Didaktik zurückgegriffen. Dabei wird angenommen, dass Kulturvergleiche dazu dienen, kulturelle Fettnäpfchen zu vermeiden, das Fremdverstehen über andere ,Kulturen' zu fördern, kulturelle Konfliktsituationen zu erklären und interkulturelle Kompetenz zu erwerben, indem Aspekte und Phänomene einer ,Kultur' einer anderen vergleichend gegenübergestellt werden. Für den Orientierungskurs kommt allerdings ein weiteres Ziel hinzu. Der Vergleich zwischen Herkunfts- und Zielkultur soll somit im Idealfall dazu führen, dass die Zielkultur positiv bewertet wird. Es deuten sich also auf der konzeptionellen Ebene des Orientierungskurses einige Spannungsmomente an. So erscheint die generelle Zielvorstellung des, mündigen Schülers' gepaart mit dem, Indoktrinationsverbot' im Konzept der politischen Bildung ebenso wie die Idee der prinzipiellen Gleichwertigkeit von Kulturen im didaktischen Konzept des Kulturvergleichs nicht widerspruchsfrei zum Anliegen des Orientierungskurses zu sein, wenn dieser sich als Angebot der Wertebildung versteht und dieses Angebot mit der normativen (und sanktionierbaren) Erwartung an die Teilnehmenden verknüpft, eine „positive Bewertung des deutschen Staates [zu] entwickeln". ${ }^{7}$ Auf welche Weise machen sich diese Spannungsmomente in der Kurssituation bemerkbar und wie werden sie von den Beteiligten bearbeitet? Welche Bedeutung erlangt dabei der Kulturvergleich? In welchen Variationen tritt er auf, wie wird also das didaktische Konzept praktisch realisiert und wie werden welche Vergleichseinheiten konstruiert und miteinander relationiert? Zur Annäherung an die hier aufgeworfenen Fragen greifen wir auf das Konzept der kommunikativen Gattung und die daraus entwickelte Methode der Gattungsanalyse zurück und verbinden dieses mit dem neuen Konzept der Invektivität.

6 Fornhoff (2018) Migration, S. $128 f$.

7 BAMF (2017) Curriculum S. 7. 
Mit dem Invektivitätsbegriff wird Herabsetzung als ein sozial strukturierender Modus von Interaktions- und Kommunikationsprozessen mit identitätsund machtpolitischer Funktion gefasst. Invektivität wird als spezifischer Modus von Interaktions- und Kommunikationsprozessen definiert, durch deren Beobachtung es erst möglich ist, die Konflikthaftigkeit, Konsensfähigkeit, Polemogenität und Reproduktion sozialer Ordnungen angemessen verstehen zu können. Es dient als Produktionsmechanismus und Transmissionsriemen sozialer In- und Exklusionsprozesse und produziert damit soziale Hierarchien und Ordnungen. ${ }^{8}$ In Bezug auf den Orientierungskurs stellt sich die Frage, in welcher Weise die oben skizzierten Spannungsmomente kommunikativ bearbeitet werden und inwieweit insbesondere im Kulturvergleich invektive Positionierungen befördert, verfestigt, zurückgewiesen oder reflektiert werden. Den Orientierungskurs betrachten wir in diesem Zusammenhang als eine didaktische Arena des kulturellen Lehrens und Lernens, in der relevantes Wissen über ,Deutschland' kanonisiert, tradiert und sozial verteilt wird. Eine didaktische Arena ist also als institutionalisierter Handlungsund Kommunikationsraum auf seine Regeln und Rahmungen hin zu untersuchen, nach denen die Distribution und Legitimation von Wissen und sozialen Positionen organisiert wird.

Im vorliegenden Beitrag konzentrieren wir uns auf die Frage, welche Formen und Funktionen der Kulturvergleich im Orientierungskurs aufweist, wie er sich koproduktiv vollzieht und welche kommunikativen Effekte sich dabei beobachten lassen. Um das invektive Potential des Kulturvergleichs und seine ordnungsbildende bzw. -transformierende Kraft zu ermitteln, bietet sich die Gattungsanalyse nach Bergmann und Luckmann an. ${ }^{9}$ Kommunikative Gattungen werden hier im Anschluss an Luckmann (1986) als gesellschaftliche Institutionen zur Vermittlung und Tradierung von handlungsorientierendem Wissen verstanden, welche die Aufrechterhaltung sozialer Ordnung stützen. In kommunikativen Gattungen sedimentiert sich kulturelles Wissen, welches sich im situativen Sprachgebrauch aktualisiert und mit sozialstrukturellem Ordnungswissen verknüpft. ${ }^{10}$ Die Untersuchung von kulturvergleichender Kommunikation und ihren positio-

8 Ellerbrock/Koch/Müller-Mall et al. (2017) Invektivität, S. 6. 9 Bergmann/Luckmann (1995) Reconstructive Genres. 10 Vgl. Luckmann (1986) Grundformen. nierenden Effekten kann daher Aufschluss über die Herstellung bzw. Transformation von gesellschaftlicher Ordnung im Orientierungskurs bieten.

Die Analyse stützt sich auf ethnografisch generiertes Datenmaterial aus vier Kursen. ${ }^{11}$ Es umfasst Beobachtungsprotokolle und Memos von 22 Unterrichtstagen, zwei Teamgesprächen, einer Abschlussfeier und einer Exkursion, 17,5 Stunden Audioaufnahmen von sechs Unterrichtstagen sowie die in den Kursen verwendeten Lehrmaterialien. Im Folgenden stellen wir zunächst unter Rückgriff auf das Konzept der kommunikativen Gattung einige theoretische und methodische Überlegungen zum kommunikativen Haushalt des Orientierungskurses an. Im zweiten Schritt werden wir der Frage nachgehen, in welchen Variationen und Kontextuierungen der Kulturvergleich im Orientierungskurs zu finden ist. Anschließend untersuchen wir inn im Hinblick auf seine außenstrukturellen Merkmale, zu denen die Didaktik des Kulturvergleichs im Fremdsprachenunterricht und ihr Niederschlag im Curriculum des Orientierungskurses als metakommunikative Rahmung ebenso gehört, wie die beziehungs- und rollenspezifischen Äußerungstypen und -rechte der Beteiligten. Sodann werden wir exemplarisch an einem Beispiel die binnenstrukturellen Merkmale kulturvergleichender Kommunikation und inre interaktive Verlaufsstruktur rekonstruieren. Abschließend diskutieren wir die Funktionen und invektiven Qualitäten des Kulturvergleichs im Orientierungskurs und fokussieren dabei besonders auf die Themenzusammenhänge, in denen kulturvergleichende Kommunikationen eingebettet sind und die Beziehungskonstellationen, die durch sie hergestellt bzw. aktualisiert werden.

\section{Kommunikative Gattungen: Theoretische und methodische Überlegungen zum kommunikativen Haushalt des Orientierungskurses}

Im Folgenden werden wir die Grundprämissen der Theorie kommunikativer Gattungen und der Gattungsanalyse kurz skizzieren und als theoreti-

11 Die Daten wurden im laufenden Forschungsprojekt Invektive Kodierungen von Interkulturalität in der Zeit von 11.2018 bis 03.2019 generiert. 
sche und methodische Heuristik für die Untersuchung typischer kommunikativer Handlungen im Orientierungskurs entfalten. In seinem programmatischen Aufsatz von 1986 Grundformen der gesellschaftlichen Vermittlung des Wissens: Kommunikative Gattungen untersucht Thomas Luckmann den Zusammenhang von Wissen, Sprache und Handeln. Wissen ist demnach für Handeln konstitutiv, es ist gesellschaftlich bestimmt und sozial verteilt. Als "subjektiv relevante Problemlösungen" 12 lagert sich Wissen in Erfahrungs- und Handlungsschemata im subjektiven Wissensvorrat ab. Man kann also in ähnlichen Situationen immer wieder auf bewährtes Wissen (etwa zur Handlungsorientierung oder -reflexion) zurückgreifen, welches damit eine handlungsentlastende Funktion erlangt. Allerdings entsteht der größte Teil des Wissens nicht durch Primärerfahrungen, sondern wird durch Sozialisation bzw. als Tradition gesellschaftlich vermittelt. Denn erst im sozialen Austausch werden subjektiv relevante Schemata zu intersubjektiven Problemlösungen. Kultur ist für Luckmann dementsprechend ein Synonym für Wissenstradition. Besondere Aufmerksamkeit lenkt er auf die Bedeutung von Sprache. Sie hat die Funktion der Objektivierung von Erfahrungsund Handlungsschemata und "kann als Ablagerung der typischen Erfahrungsschemata, die in einer historischen Gesellschaft von Bedeutung sind, aufgefaßt werden". ${ }^{13}$ Diese intersubjektiv geteilten, in kommunikativen Mustern verfestigten und institutionalisierten Erfahrungs- und Handlungsschemata nennt Luckmann kommunikative Gattungen. Für die sozial- und kulturwissenschaftliche Forschung sind sie, dem Autor folgend, ein zentraler Untersuchungsgegenstand, da sie als "sinnstiftende und handlungsorientierende Innenarchitektur einer Gesellschaft"14 einen induktiven Zugang zu den kulturell, sozial und historisch spezifischen Ordnungsstrukturen von Gesellschaften, aber auch kleineren sozialen Entitäten, wie Organisationen, Subkulturen oder Berufskulturen bietet.

Mit "Verfestigung" und "Formalisierung" beschreiben Günthner und Knoblauch "die wesentlichen Merkmale kommunikativer Gattungen." 15

12 Luckmann (1986) Grundformen, S. 199.

13 Luckmann (1986) Grundformen, S. 200.

14 Knoblauch/Luckmann (2003) Gattungsanalyse, S. 546. 15 Günthner/Knoblauch (1994) Gattungen, S. 702.
Sie unterscheiden zwischen vollausgereiften und nicht vollausgereiften Gattungen, mit je unterschiedlichen Komplexitäts- und Formalisierungsgraden. ${ }^{16}$ So weist etwa eine Begrüßung zwischen Bekannten, die sich zufällig auf der Straße treffen, einen geringeren Komplexitäts- und Formalisierungsgrad auf, als die Eröffnung eines Gerichtsverfahrens, das einem vorgeschriebenen Ablauf zu folgen hat. Analytisch lassen sich kommunikative Gattungen in Außenstruktur, Binnenstruktur und situative Realisierungsebene unterteilen. In der Außenstruktur bildet sich die Beziehung zwischen kommunikativem Handeln und Sozialstruktur ab. Daher werden auf dieser Ebene zunächst die Teilnehmerkonstellationen und ihre je spezifischen Rahmen in den Blick genommen. Hierzu zählen erstens, institutionelle Rahmungen, im Fall des Orientierungskurses etwa die Trägerstruktur, die den Kurs eng an die Integrationspolitik der Bundesregierung bindet und ihr unterstellt; ${ }^{17}$ zweitens, soziale Konventionen und Normen, die rollen- und positionsadäquate Sprachhandlungen, Beteiligungsrechte und -pflichten regeln, aber auch die konstellationsspezifische soziale Verteilung von Wissen. So haben in einem Orientierungskurs Lehrkräfte andere Rechte und Pflichten als Kursteilnehmende. Aber auch unter Letzteren sind, abhängig von ihrem ausländerrechtlichen Status, Rechte und Pflichten ungleich verteilt. Hier werden etwa Teilnahmeberechtigte von Teilnahmeverpflichteten unterschieden. Die Konstellationen differieren ferner im Hinblick auf die soziale Verteilung des (sprachlichen, kulturellen) Wissens, das für den Kurs maßgeblich ist. Im Kurs sind die deutsche Sprache und spezifisches Wissen über Deutschland als Lernziele definiert, welches die Lehrkraft an die Teilnehmenden vermitteln soll, die insofern als Wissensautorität gelten kann, während das mitgebrachte, sprachliche, kulturelle und Erfahrungswissen der Teilnehmenden für den Kurserfolg irrelevant ist. Die Kurse sind zudem meist sehr heterogen im Hinblick auf die Sprachen, Wissenstraditionen und die formale Bildung der Teilnehmenden.

16 Vgl. Günthner/Knoblauch (1994) Gattungen, S. 703. 17 So sind Curriculum, Lehrmaterialen, die Befugnis, als Sprachschule Integrationskurse anzubieten und in ihnen zu lehren, durch zentrale Zulassungsverfahren geregelt, welche im Zuständigkeitsbereich des BAMF liegen. 
Die Binnenstruktur umfasst jene sprachlichen Phänomene, die eine kommunikative Gattung als solche in ihrem performativen Akt intersubjektiv erkennbar machen. Hierzu zählen prosodische und verbale, mimische und gestische Elemente und ihre Verwendung in "Worten und Phrasen, rhetorischen Figuren und Tropen."18 Für den Orientierungskurs ist die Kombination von schriftlich-verfassten und visuell angereicherten Aufgabenstellungen im Lehrbuch und den ergänzenden Lehrmaterialien einerseits mit dem mündlichen Unterrichtsgespräch andererseits charakteristisch. Erstere weisen einen hohen Formalisierungsgrad auf und orientieren sich sehr eng an den Prüfungsaufgaben des Tests ,Leben in Deutschland', auf die auch regelmäßig im Lehrbuch verwiesen wird. Das Unterrichtsgespräch lässt der Lehrkraft demgegenüber in didaktischer wie thematischer Hinsicht einigen Spielraum. Sie kann eigene Schwerpunkte setzen und die in den Lehrmaterialien offerierten Inhalte selektiv bearbeiten und zusammenfügen und mit eigenem Zusatzmaterial ergänzen. Die schriftlichen Lehrmaterialien weisen einige Gesamtmuster auf. Hierzu zählen u.a. die stilistische Figur der Schlagzeile in Übungsaufgaben, mit denen auf aktuelle gesellschaftliche Debatten Bezug genommen werden soll, evaluative Fragen (z.B.: "Wie gut kennen Sie die Grundrechte?"), inhaltliche Verfestigungen von Unterscheidungen ,ausländisch/deutsch' durch wiederholte Bezugnahmen auf bestimmte Figuren (deutsche Familien/ ausländische Personen) sowie Stereotypen, die in einer journalistischen Darstellungsform verfasst sind. Zu den binnenstrukturellen Merkmalen des Unterrichtsgesprächs zählen u.a. das von der Lehrkraft angeleitete kollaborative Lesen der Übungsaufgaben, mit dem sprachliches Verständnis gesichert werden soll, das korrektive Feedback auf Äußerungen der Kursteilnehmenden und die Verwendung bestimmter Fragetypen, die wir in Abschnitt 4 näher betrachten werden. ${ }^{19}$ Die situative Realisierungsebene adressiert schließlich das konkrete raumzeitlich situierte Ereignis

18 Luckmann (1992) Rekonstruktive Gattungen, S. 39. 19 In der Analyse der Unterrichtsgespräche werden im vorliegenden Beitrag prosodische, mimische und gestische Elemente nicht berücksichtigt, da für die ausgewählten Sequenzen keine Aufzeichnungen vorliegen und sich die Analyse allein auf Beobachtungsprotokolle und die Lehrmaterialen stützt. in dem eine kommunikative Gattungen interaktiv und sequenziell realisiert wird. Sie ist der eigentliche Fokus der Analyse, denn erst in ihrer situativen Realisierung wird eine kommunikative Gattung koproduktiv hergestellt und die sie konstituierende Sozialbeziehung der Beteiligten als Schnittstelle zwischen kommunikativer Handlung und Sozialstruktur aktualisiert.

Der Orientierungskurs ist gattungstheoretisch in mehrfacher Hinsicht ein interessanter Untersuchungsgegenstand. Er ist erstens ein Ort, an dem sich die für die Gegenwartsgesellschaft repräsentative sprachliche, ethnische, kulturelle und soziale Heterogenität abbildet. Im Orientierungskurs treffen einander individuell meist fremde Angehörige unterschiedlicher Sprach- und Kulturgemeinschaften aufeinander. Sie bilden in sozialer und kultureller Hinsicht heterogene Konstellationen, knüpfen wechselseitig an verschiedene, häufig unbekannte Verhaltensweisen und kulturelle Muster an. Jeder Kurs muss sich also selbst zunächst einmal als soziale Gruppe formieren und sich auf eine gemeinsame Unterrichtspraxis verständigen. Der Orientierungskurs kann in dieser Hinsicht als Gattungsschmiede betrachtet werden. Er stellt gleichzeitig selbst eine gesellschaftliche Institution zur Vermittlung und Tradierung von handlungsorientierendem Wissen dar. Er kann folglich daraufhin untersucht werden, welches kulturelle Wissen für eine selbstständige Lebensführung in Deutschland als maßgeblich erachtet und durch staatlich geregelte Zulassungs- und Prüfungsverfahren kanonisiert wird und wie dieses Wissen mit der im Kurs anwesenden Vielfalt an Wissenstraditionen vermittelbar ist. Schließlich greift der Orientierungskurs bei der Wissensvermittlung auf ein Repertoire an kommunikativen Mustern zurück, die ihrerseits gattungsanalytisch auf ihre Problemlösungsfunktionen hin untersucht werden können: Welche kommunikativen Probleme werden in diesen heterogenen Konstellationen von den Beteiligten bearbeitet? Welcher verfestigten Problemlösungsmuster bedienen sie sich dabei und auf welche Ordnungsstrukturen und normativen Orientierungen lassen die im Orientierungskurs verwendeten kommunikativen Gattungen schließen? Dies sind die Leitfragen unserer gattungsanalytischen Untersuchung des Orientierungskurses. Für den vorliegenden Beitrag konzentrieren wir uns zunächst auf das mit Abstand am häufigsten vorkommende kommuni- 
kative Muster des Kulturvergleichs, welches wir im Folgenden auf seine Formen und Funktionen untersuchen werden. In welchen thematischen Zusammenhängen taucht der Kulturvergleich im Orientierungskurs auf? Was ist überhaupt Gegenstand des Vergleichs und wie sind die zu vergleichenden Einheiten einander zugeordnet? Welche Teilnehmerkonstellationen werden in seinem sequenziellen Ablauf hergestellt? Es geht also einerseits um die Frage, welche als kulturell zu vergleichenden Einheiten auf welche Weise miteinander relationiert werden und sich dadurch wechselseitig Bedeutung zuweisen, und andererseits darum, wie die Teilnehmenden über die Praxis des Kulturvergleichs sich zueinander relationieren und positionieren. Wir fassen zunächst den Stand der Diskussion zum Kulturvergleich in der Fremdsprachendidaktik zusammen und fragen, welche Konzepte der Didaktik des Kulturvergleichs im Curriculum des Orientierungskurses aufgegriffen werden. Sodann beginnen wir mit einer deskriptiven Analyse der Variationen kulturvergleichender Kommunikation im Orientierungskurs. Der Kulturvergleich ist, wie eingangs angedeutet, als didaktische Methode für den DaZ- und DaF-Unterricht konzipiert und findet sich implizit auch im Curriculum des Orientierungskurses. In den Lehrmaterialen gibt es dementsprechend zahlreiche Übungen, die zum Vergleich zwischen Herkunfts- und Zielkultur bzw. den jeweils im Kurs repräsentierten Nationalkulturen auffordern. Noch häufiger finden wir spontane Kulturvergleiche in den Unterrichtsgesprächen, selbst dort, wo die Aufgabenstellung aus den Lehrmaterialien diese gar nicht vorsehen. Wir werden uns daher im zweiten Teil der Analyse auf diese spontanen Kulturvergleiche konzentrieren.

\section{Variationen und Kontexturierungen des Kulturvergleichs im Fremdsprachenunterricht}

Bevor wir auf den kulturvergleichenden didaktischen Ansatz im Orientierungskurs eingehen, sollen die unterschiedlichen Ansätze im Fremdsprachenunterricht erläutert werden. Nach der Klassifizierung von Günter Weimann und Wolfram Hösch gibt es im Fremdsprachenunterricht drei didaktische Ansätze der Kulturvermittlung: den kognitiven, den kommunikativen und den interkulturellen Ansatz. ${ }^{20} \mathrm{Zu}$ beachten ist hierbei, dass diese drei Ansätze in der Praxis selten in reiner Form vorkommen, sondern eher in einer gemischten Form zu finden sind. ${ }^{21}$ Als gemeinsames didaktisches Lernziel der Kulturvermittlung gilt demnach das Verständnis der Zielkultur zu fördern, die Ansätze unterscheiden sich lediglich darin, welche Ebenen des Wissens sie dabei fokussieren. Der kognitive Ansatz zielt auf "die Aneignung von Wissen, Fakten und Daten über ein Land"22 ab.

Das Ziel des kommunikativen Ansatzes ist

die sprachliche und kulturelle Handlungsfähigkeit in der Zielsprache und Zielkultur und die Entwicklung von Einstellungen wie Offenheit, Toleranz und Kommunikationsbereitschaft gegenüber der Zielkultur. Landeskunde in diesem Verständnis will vor allem das Gelingen sprachlicher Handlungen im Alltag und das Verstehen alltagskultureller Phänomene unterstützen. Sie ist sowohl informationsbezogen als auch handlungsbezogen konzipiert. Sie vermittelt also Wissen über die fremde Kultur als eine wesentliche Voraussetzung adäquater Sprachverwendung. ${ }^{23}$

Das Wissen über die Zielkultur ist genauso ein wichtiges Lernziel des interkulturellen Ansatzes, allerdings geht der interkulturell ausgerichtete Fremdsprachenunterricht darüber hinaus und zielt nicht nur darauf ab, Informationen zu vermitteln. ${ }^{24}$ Dieser Ansatz geht auf die Theorien von Alexander Thomas und Geert Hofstede zurück. Demnach sollen durch den didaktischen Kulturvergleichsansatz „interkulturelle Informationsdefizite, Dominanz und Überlegenheitsintentionen, Bedrohungsängste, Vorurteile und destruktive nationale und kulturelle Stereotypisierungen, Fremdenfeindlichkeit abgebaut werden". ${ }^{25}$

Auch wenn die Ansätze unterschiedliche Akzente bei der Frage der Beschaffenheit des kulturellen Wissens setzen, folgen sie der gemeinsamen Überzeugung, dass beim Fremdspracherwerb auch ein Bewusstsein bzw. Wissen über kulturelle Differenzen erlangt und ein tieferes Verständnis

20 Vgl. Weimann/Hösch (1993) Kulturverstehen.

21 Vgl. Zeuner (2017) Landeskunde, S. 8.

22 Zeuner (2017) Landeskunde, S. 11.

23 Zeuner (2017) Landeskunde, S. 1

24 Vgl. Zeuner (2017) Landeskunde, S. 11.

25 Thomas (1993) Kulturvergleichende Psychologie, S. 378. 
für die eigene im Verhältnis zur fremden Kultur entwickelt werden sollte. Der Kulturvergleich steht hier notwendigerweise im Zentrum, wobei er unter der Maßgabe der Wertfreiheit und Gleichwertigkeit von Kulturen geschehen soll. Diese normative Positionierung wird in expliziter Abgrenzung zur Geschichte des ethnologischen Kulturvergleichs vorgenommen, welche bereits in den 1980er und 1990er Jahren wissenschaftskritisch auf ihre ethnozentrischen Vorannahmen und invektiven Effekte befragt worden war. ${ }^{26}$ Die Ziele des Kulturvergleichs im Fremdsprachenunterricht werden vor diesem Diskurshintergrund von den Anliegen des ethnologischen Kulturvergleichs ausdrücklich unterschieden. Letzterer sei im Wesentlichen durch koloniale Perspektiven auf vermeintlich primitive Kulturen und auf von Forschungsreisenden neu ,entdeckte Regionen' geprägt. ${ }^{27}$ Das Vergleichsverfahren in der Ethnologie ziele darauf $a b$, die "Variationsbreite menschlichen Verhaltens" 28 zu bestimmen und nach allen möglichen "denkbaren Verhaltensmöglichkeiten in menschlichen Gesellschaften"29 zu suchen. Sie würden auch verwendet, um "Typologien und Klassifikationen von Gesellschaftsformen und Kulturmerkmalen zu erstellen." 30 Der didaktische Kulturvergleich im interkulturellen Ansatz gilt demgegenüber als eine einfache und zugängliche Methode für die Lernenden, um Bezüge zwischen deren Lebenswelten und der ,fremden Kultur' herzustellen. Dennoch gilt es auch für die Kulturdidaktik im Fremdsprachenunterricht als zwingend, sich in Grundzügen mit den kritischen Diskursen über den wissenschaftlichen Kulturvergleich zu beschäftigen. ${ }^{31}$

Kulturvergleich definiert Adelheid $\mathrm{Hu}$ als einen Vergleich zwischen Phänomenen der eigenen, bekannten, nachvollziehbaren und der neuen, fremden Kultur. Sie stellt fest, dass er als kog-

26 Unter dem Stichwort der „Krise der ethnographischen Repräsentation" (Berg/Fuchs [1993] Kultur) wurde die Funktion und Rolle der Ethnologie für die europäische Expansion und die Etablierung kolonialer Machtverhältnisse kritisch reflektiert, das Verhältnis von ethnografischer Autor*innenschaft und Autorität hinterfragt (Clifford/Marcus [1986] Writing Culture) und letztlich der Kulturbegriff selbst auf den Prüfstand gestellt (Abu-Lughod [1991] Writing Against Culture).

27 Vgl. Chen (2013) Das Eigene, S. 52.

28 Bollig (2003) Interkulturelle Vergleichsverfahren, S. 395.

29 Bollig (2003) Interkulturelle Vergleichsverfahren, S. 395.

30 Chen (2013) Das Eigene, S. 53.

31 Vgl. Chen (2013) Das Eigene, S. 55f. nitiver Prozess im Kontext des Fremdsprachenunterrichtes ständig auftritt und dabei oft mit Wertungen verbunden sei. ${ }^{32}$ Chen problematisiert in diesem Zusammenhang, der Kulturvergleich würde unbewusst und ungewollt zu einem Werkzeug der gegenseitigen Abgrenzung. ${ }^{33}$ Die ethische Position der Landeskundedidaktik zur Kulturvermittlung im Fremdsprachunterricht steht offenbar in einem gewissen Widerspruch zu seiner Praxis. Dieser Widerspruch verweist auf ein Spannungsverhältnis zwischen kulturellen und sozialen Zugehörigkeiten, mithin auf eine "Asymmetrie ,ethno-natio-kultureller Zugehörigkeiten' (Mecheril 2003) in einem globalen Machtgefüge, in dem die nationalstaatliche Zugehörigkeit über die Anerkennungswürdigkeit als Rechtssubjekt [...] entscheidet". ${ }^{34}$ An der historischen Entwicklung dieser Asymmetrie im Kontext des europäischen Kolonialismus und ihrer wissenschaftlichen Legitimation etwa in der Rassentheorie oder im Modernisierungsparadigma hatte die Ethnologie zweifelsohne ihren Anteil. Diese invektiven ,Wissenstraditionen' wirken bis heute in der Wahrnehmung und Bewertung des Anderen im Horizont des Eigenen fort, auch wenn sie in den Wissenschaften längst als illegitim gelten. Die sozialstrukturellen Dimensionen nationalkultureller Verhältnisse werden im Konzept der Interkulturalität und des didaktischen Kulturvergleichs bislang weitgehend ausgeblendet. ${ }^{35}$ Dies wird auch in der Problematik deutlich, ein für den Kulturvergleich adäquates Tertium Comparationis zu finden. Das Dritte des Vergleichs, welches die Basis des Kulturvergleichs bildet, weil erst dadurch definiert wird, in welcher Hinsicht die zu vergleichenden Einheiten als vergleichbar angenommen werden, werde, so $\mathrm{Hu}$, meist als ortlos angenommen, so als könne man von hier aus unbeteiligt und objektiv Kulturen miteinan-

$32 \mathrm{Vgl}$. Hu (2010) Kulturvergleich, S. 178.

33 Vgl. Chen (2013) Das Eigene, S. 58.

34 Ott (2020) Arbeitspapier, S. 12f.

35 In den letzten Jahren mehren sich kritische Perspektiven auf die interkulturelle Landeskunde und deren normative Verortung im Fremdsprachunterricht. Es wird eine grundlegende Neuausrichtung der Kulturdidaktik gefordert, diese solle sich an neuen Ansätzen wie der "diskursiven Landeskunde" nach Altmayer oder dem kulturreflexiven Lehren und Lernen nach Haase und Höller orientieren.; Haase/Höller (2017) Kulturelles Lernen; Dirim/Wegner (2018) Normative Grundlagen. 
der vergleichen. ${ }^{36}$ Zusammenfassend kann konstatiert werden, dass dem Kulturvergleich durch seine historische Verwendung in der Ethnologie sowie durch die Spannungen zwischen kulturellen und sozialstrukturellen Zugehörigkeitsordnungen ein invektives Potential innewohnt. Dieses wird in der Fremdsprachendidaktik einseitig mit Blick auf die Invektivgeschichte des ethnologischen Kulturvergleichs in ein normatives Wertfreiheitspostulat aufgelöst, welches in der Praxis jedoch an seine Grenzen gerät. Schauen wir uns nun an, auf welche Weise die Didaktik des Kulturvergleichs im Orientierungskurs umgesetzt wird.

\section{Der Kulturvergleich im Curriculum des Orientierungskurses}

Der Orientierungskurs ist inhaltlich und didaktisch nach den folgenden Leitlinien konzipiert. Er soll erstens die Grundprinzipien der politischen Bildung nach dem Beutelsbacher Konsens ${ }^{37}$ berücksichtigen. Zweitens sollen die Inhalte mit den jeweiligen regionalen oder kommunalen Besonderheiten der Orte, an denen die Teilnehmenden aktuell leben, vermittelt werden. Die Themenbereiche im Unterricht sollen also "konkrete lokale Bezüge herstellen, weil die Integration vor allem auf der kommunalen Ebene stattfindet". ${ }^{38}$ Schließlich soll in den Kursen auch Bezug auf aktuelle gesellschaftliche Migrations- und Integrationsdiskurse genommen werden, in welchen

echte oder auch nur scheinbare Gegensätze zwischen den Gepflogenheiten in Deutschland auf der einen und in den Herkunftsländern auf der anderen Seite hergestellt [werden]. Beispielhaft zu nennen sind hier

$36 \mathrm{Vgl.} \mathrm{Hu} \mathrm{(2010)} \mathrm{Kulturvergleich,} \mathrm{S.} 178$.

37 Demnach soll politische Bildung als Zielvorstellung die "Mündigkeit des Schülers" zugrunde legen, woraus sich ein Indoktrinationsverbot ebenso ergebe, wie ein offener Umgang mit politisch und wissenschaftlich kontroversen Themen. Ziel der politischen Bildung ist entsprechend zur Analyse einer politischen Situation zu befähigen und "nach Mitteln und Wegen zu suchen, die vorgefundene politische Lage im Sinne seiner Interessen zu beeinflussen" (Wehling 1977, S. 179f.), zit. nach Sutor (2011) APuZ B 45/2002, unter https://www.bpb.de/die-bpb/51310/beutelsbacherkonsens (letzter Zugriff: 22.10.2021).

38 BAMF (2017) Curriculum, S. 14.
Themen wie Kopftuch- oder Burkadebatte, Zwangsheirat, Gewalt in der Familie, Ehrenmord etc. ${ }^{39}$

Zusammenfassend soll der Orientierungskurs also nicht nur ein lebensweltnahes Angebot der politischen Bildung an Migrierte sein, er soll die Teilnehmenden auch mit Topoi vertraut machen, von denen sie „unter Umständen selbst [...] betroffen sein können" 40 . Dabei bleibt offen, worauf sich die mögliche Betroffenheit bezieht. Antizipiert das Curriculum, die Teilnehmenden könnten selbst diese Topoi als kulturelle Praxen mitbringen und etwa Ehrenmorde begehen oder möglicherweise Opfer einer solchen Gewalttat werden? Oder sollen sie darauf vorbereitet werden, dass sie von den negativen Effekten dieses Diskurswissens betroffen sein könnten, welches über Migrierte zirkuliert?

Neben den didaktischen Leitlinien, in denen sich noch kein expliziter Hinweis auf die Didaktik des Kulturvergleichs findet, sind im Curriculum auch die Ziele des Orientierungskurses festgelegt. Die Teilnehmenden sollen demnach u.a. Verständnis und eine positive Haltung zum deutschen Staatswesen entwickeln, sie sollen Kenntnis über die Rechte und Pflichten als Einwohner und Staatsbürger erlangen, zur Teilhabe am gesellschaftlichen Leben befähigt werden und interkulturelle Kompetenz erwerben. ${ }^{41}$ Damit ist insbesondere gemeint, dass die Kursteilnehmenden zur Reflexion über interkulturelle Unterschiede und Gemeinsamkeiten zwischen Deutschland und ihren Herkunftsländern angeregt werden sollen, um Bezüge zwischen beiden Kulturen herzustellen und die eigenen moralischen Orientierungen und Wissenstraditionen vor dem Horizont der Zielkultur neu zu bewerten. Der Vergleich zwischen Ziel- und Herkunftskultur wird damit ins Zentrum des interkulturellen Lernens gerückt und in den Lehrmaterialien durch entsprechende Aufgabenstellungen etabliert. Je nach Unterrichtssituation variieren Formen und Verfestigungsgrade kulturvergleichender Kommunikation, wir unterscheiden daher die im Lehrbuch formalisierten von den im Unterrichtsgespräch spontan stattfindenden Kulturvergleichen.

39 BAMF (2017) Curriculum, S. 15

40 BAMF (2017) Curriculum, S. 15

41 BAMF (2017) Curriculum, S. 7. 


\subsection{Formalisierter Kulturvergleich: Kulturvergleichende Übungen in den Lehrmaterialien}

Im Lehrbuch gibt es zahlreiche Übungen, die zum Vergleich zwischen Herkunfts- und Zielkultur bzw. den jeweils im Kurs repräsentierten Nationalkulturen explizit oder implizit auffordern. Wie an den folgenden Beispielen aus zwei durch das BAMF für den Orientierungskurs zugelassenen Lehrbüchern 100 Stunden Deutschland und Orientierungskurs zu sehen ist, sind die kulturvergleichenden Übungen formal sehr ähnlich aufgebaut: "Sammeln Sie in der Gruppe Unterschiede und
Gemeinsamkeiten in den Systemen in ihren Heimat-
ländern!"42

„Vergleichen Sie die Situation in Deutschland mit Ihrem Heimatland. Welche Rechte haben Frauen und Männer? Wo gibt es Unterschiede und Gemeinsamkeiten?"43

„Kennen Sie andere politische Systeme? Was wissen Sie über diese Systeme?"44

Sie sind entweder als Entscheidungs- oder Bestätigungsfragen formuliert, die den Kursteilnehmenden keine offene Antwortmöglichkeit anbietet, als Imperativsatz, der die Kursteilnehmenden zu einer Handlung auffordert oder als Was-Konstruktion, die dazu dient die mögliche Antwort auf eine vorgängige geschlossene Frage "Ja", "Nein" oder "Weiß nicht" zu vertiefen und zu erklären. Das Aufgabenziel legt den Fokus auf ein spezifisches Thema bzw. Phänomen als Tertium Comparationis, welches in Gruppenarbeit verglichen und danach im Unterricht präsentiert werden soll. Das Dritte des Vergleichs ist in der Aufgabe meist klar formuliert und vorgegeben, z.B. 'Frauenrechte', ,Politische Systeme' oder ,Umgang mit der Zeit'. Während der Gruppenarbeit beobachtet die Lehrperson die Kursteilnehmenden, wie sie miteinander agieren und greift $a b$ und zu korrigierend ein oder beantwortet die Fragen der Kursteilnehmenden. Auffällig ist, dass die im Lehrbuch definierten Aufgabenstellungen im Unterricht eine Transformation erfahren. Sie werden selten so bearbei-

42 Butler/Würtz/Kotas et al. (2017) 100 Stunden Deutschland, S. 19.

43 Schote (2017) Orientierungskurs, S. 73.

44 Butler/Würtz/Kotas et al. (2017) 100 Stunden Deutschland, S. 19. tet, wie es das Lehrbuch vorsieht, sondern von der Lehrkraft situativ abgewandelt, so dass sie sich gut in den laufenden Unterrichtsprozess einfügen. Dabei kommt es erstens häufig zum spontanen Einsatz kulturvergleichender Fragen auch dort, wo sie im Lehrbuch nicht vorgesehen sind. Wenn im Lehrbuch dazu aufgefordert wird, Unterschiede und Gemeinsamkeiten zwischen Herkunfts- und Zielkultur bzw. zwischen verschiedenen Ländern zu sammeln, wird zweitens im Unterrichtsgespräch meist auf die Unterschiede fokussiert, wie sich in der nachfolgenden Analyse zeigen wird.

\subsection{Spontane Kulturvergleiche im Unterricht}

Neben diesen im Lehrmaterial formalisierten kulturvergleichenden Aufgabenstellungen ist damit der ,spontane' Kulturvergleich eines der am häufigsten gebrauchten kommunikativen Muster im Orientierungskurs. ${ }^{45} \mathrm{Er}$ zeichnet sich dadurch aus, dass er im Unterrichtsgespräch entweder von Teilnehmenden oder Lehrkräften initiiert wird, ohne dass die Aufgabenstellung dies vorsieht. Insbesondere der von den Lehrkräften initiierte spontane Kulturvergleich weist unabhängig von der jeweiligen Lehrkraft starke Ähnlichkeiten auf. Er wird in der Regel mit einer Frage eingeleitet, welche dazu auffordert einen bestimmten Sachverhalt, einen Wert oder eine Norm zwischen den durch die Kursteilnehmenden repräsentierten Ländern zu vergleichen. Die wichtigsten sind WieKonstruktionen (z.B. "Wie ist das in X-Land?"), Was-Konstruktionen (Z.B. "Was ist mit X?") sowie Entscheidungs- oder Bestätigungsfragen, die mit "Gibt es das in ..." beginnen. Während WieKonstruktionen im Prinzip einen weiten Raum für unterschiedliche Antwortmöglichkeiten zulassen, werden mit der geschlossenen Frage "Gibt es das in ...?" "spezifische typ-konforme Antworten"46 wie "Ja", "Nein" oder "Weiß nicht" nahegelegt. Das Pronomen ,das' bezieht sich dabei auf den Sachverhalt, ein Phänomen, eine Norm oder einen Wert, über den in dem Moment im Kurs diskutiert wird bzw. der von der Lehrkraft zuvor

45 Die häufigsten kulturvergleichenden Fragen im Orientierungskurs wurden durch eine lexikalische Suche in den ca. $70 \%$ der generierten erhobenen Daten im MAXQDA erfasst. 46 Zit. nach Betz (2017) Diskursmarker, S. 193. 
als Bezugsreferenz eingeführt wurde. Die Frage setzt voraus, dass der kommunizierte Sachverhalt im Heimatland der Kursteilnehmenden auch von Relevanz ist und dass er sich als, anders' oder ,fremd' darstellen und mit der Zielkultur vergleichen lässt. Durch die Art der Frage zeigt die Lehrkraft an, dass sie keine nähere Erklärung erwartet, sondern entweder eine Bestätigung oder eine Negation über den kommunizierten Sachverhalt. Demgegenüber fordern Was-Konstruktionen die Kursteilnehmende zur Erklärung auf, um eine differenzierte Betrachtung über den kommunizierten Sachverhalt zu erhalten. Zu beachten ist, dass die Antworten der Kursteilnehmende auf die spontanen kulturvergleichenden Fragen nicht immer von Relevanz sind. Dies ist auf das vorausgesetzte Wissen der Interagierenden zurückzuführen, dass es auf die gestellten Fragen erwartbare Antworten gibt. Allgemein gilt: „W-Fragen projizieren über das Fragewort, was eine passende Antwort ist. Darüber hinaus enthalten W-Fragen auch Präsuppositionen, zu denen Antwortende Stellung nehmen können." 47 Im Gegensatz zu formalisierten Kulturvergleichen im Lehrbuch ist das Tertium Comparationis bei den spontan kulturvergleichenden Fragen nicht immer eindeutig und kann durch die Beteiligten in eine andere Richtung gelenkt werden oder auch ungeklärt bleiben. Wir werden im Folgenden anhand einer exemplarischen Unterrichtssequenz die situative Realisierung einer kulturvergleichenden Kommunikation im Orientierungskurs auf ihre Grundstruktur und ihren interaktiven Ablauf untersuchen. Von Interesse ist dabei erstens die Beschaffenheit des Tertium Comparationis sowie der Vergleichseinheiten, die an ihm gebildet und miteinander relationiert werden.

\subsection{Grundstruktur und interaktiver Ablauf des spontanen Kulturvergleichs}

Die im Folgenden zu analysierende Sequenz stammt aus einem Unterrichtsgespräch des zweiten Tages eines Orientierungskurses in einer Sprachschule. ${ }^{48}$ Der Kurs hat acht Teilnehmende und wird von einer Dozentin geleitet, die wir Nancy

47 Betz (2017) Diskursmarker, S. 193.

48 Beobachtungsprotokoll - OK 3 - beobachtet am 07.12.2018. nennen wollen. ${ }^{49}$ Die vier Männer und vier Frauen im Alter zwischen 19 und 60 Jahren kommen u.a. aus Russland, Eritrea, Sudan, Vietnam und Iran. Alle, bis auf die Männer aus dem östlichen Afrika und Iran sind Migrant*innen mit einem sicheren Aufenthaltsstatus. Die Beteiligten kennen einander nicht aus vorherigen Kursen. Bei der vorliegenden Sequenz handelt es sich um die Fortsetzung eines Themas vom Vortag. Es geht um die Vielfalt an Lebensformen in Deutschland, die Übung ist Teil des Moduls Mensch und Gesellschaft. Nancy wiederholt zunächst noch einmal die Antwort auf die Frage des Vortags, die sie an die Tafel zeichnet: "Was ist eine traditionelle Familie?" Demnach hätten viele sich die traditionelle Familie so vorgestellt:

$$
\text { Mann + Frau + Kinder }
$$

Nancy bemerkt dann, dass es in Deutschland noch andere Familienformen gäbe und fordert die Teilnehmenden mit der Frage "Welche Formen gibt es noch in Deutschland?" auf, diese zu benennen. Die Teilnehmenden kommen dieser Aufforderung nach und nennen verschiedene Familienformen, die von Nancy sprachlich korrigiert als "gleichgeschlechtliche Beziehung/Paare mit/ohne Kind" an der Tafel festgehalten werden. Sie beantwortet die Frage des Teilnehmers Hasib nach der Bedeutung des Worts, gleichgeschlechtlich' und fordert dann die Teilnehmenden dazu auf, darüber nachzudenken, wie es dazu gekommen ist, dass sich in Deutschland die Familienformen diversifiziert haben, "warum" also, "dieses klassische Bild anders geworden ist in Deutschland". Eine Nachfrage des Teilnehmenden Hasib und seine darauffolgende moralisch-wertende Äußerung bezüglich der in Deutschland verbreiteten Praxis nichtehelicher Lebensgemeinschaften ("Nur Freunde, das ist falsch.") wird von Nancy als „nicht verstanden" kommentiert und von Hasib daraufhin nicht weiterverfolgt. Nancy kommt stattdessen zu ihrer Frage zurück, die sie nun selbst an der Tafel beantwortet: Demnach erodierte das traditionelle Familienmodell aufgrund veränderter gesellschaftlicher (und rechtlicher) Normen, die es erstens Frauen ermöglicht, selbstbestimmt einer Erwerbstätigkeit nachzugehen und die es zweitens verheirateten Paaren erlaubt, sich zu trennen. Nun initiiert Hasib eine

49 Alle hier verwendeten Namen sind Pseudonyme. 
kulturvergleichende Kommunikation, die allerdings keine Resonanz findet: Er kommt auf die Frage des nichtehelichen Zusammenlebens zurück und möchte zunächst genauer wissen, wie weit diese Lebensform in Deutschland verbreitet ist. Seine Frage nach dem prozentualen Anteil an nicht-verheirateten Paaren kann Nancy nicht beantworten. Hasib wiederholt seine zuvor bereits geäußerte moralische Wertung nichtehelicher Lebensgemeinschaften, relativiert sie jedoch zugleich, in dem er die in Deutschland gültige Normalität von der in muslimischen Ländern gültigen Normalität unterscheidet: „In muslimischen Ländern, das ist falsch. Hier Mädchen und Jungen zusammenleben, aber in muslimischen Ländern nicht."

An dieser Stelle des Unterrichtsgesprächs dient der seitens eines Kursteilnehmers initiierte Vergleich dazu, die Differenz zwischen normativen bzw. moralischen Orientierungen zu erfassen und inre jeweilige Gültigkeit einzugrenzen. Das Tertium Comparationis, dasjenige Kriterium, welches die zu vergleichenden Einheiten vergleichbar macht, ist hier nicht, wie in der Übung angelegt, Formen des Zusammenlebens allgemein zu behandeln, sondern die Frage, wie und mittels welcher Instanzen Lebensformen ihre soziale Einbettung und Anerkennung erfahren (sollen). Als Einheiten des Vergleichs unterscheidet Hasib Deutschland von muslimischen Ländern. Er relationiert also einen einzelnen Nationalstaat und dessen angenommene kulturelle Verfasstheit mit einer unbestimmten Anzahl an Ländern, die sich seines Erachtens nach im Gegensatz zu Deutschland nicht über eine Nationalkultur, sondern über religiöse Wissenstraditionen definieren und in dieser Hinsicht als gleich angenommen werden können. ${ }^{50}$

Wie zuvor bleibt auch dieser Redebeitrag von Hasib sozial folgenlos, insofern niemand daran

50 Wir können hier nicht mit Sicherheit sagen, ob die Verwendung des Plurals "muslimische Länder" beabsichtigt ist oder Hasib hier Plural mit Singular verwechselt. Er verwendet auch an anderer Stelle "muslimische Länder" in einem Satz im Zusammenhang mit seinem Herkunftsland, in dem der Gebrauch des Singulars naheliegender wäre. Abgesehen davon, dass die Intention des Sprechers nicht allein ausschlaggebend für den sozialen Sinn einer Äußerung ist, könnte die Verwechslung von Singular und Plural, so sie denn vorliegt, dafürsprechen, dass "muslimische Länder" eine für Hasib eingängigere Vokabel ist, als „muslimisches Land"; die Verallgemeinerung des Muslimischen durch die Verwendung des Plurals hier also offenbar eine diskursive Realität widerspiegelt, in welcher Hasib Deutsch lernt. anschließt. Auch Nancy reagiert nicht auf inn, sondern wendet sich an eine andere Teilnehmerin, die unmittelbar im Anschluss an Hasib eine Frage stellt und wissen möchte, ob in Deutschland Männer mehrere Frauen heiraten dürfen. Nancy beantwortet die Frage, und gibt an, dass dieser Fall sehr selten in Deutschland sei und die Deutschen eher monogam lebten. Sie beendet dann den mündlichen Austausch und setzt das Thema mit einer Übung aus dem Lehrbuch fort. Unter der Überschrift "Wie leben die meisten Menschen in Deutschland zusammen?" sind verschiedene Bilder zu sehen, die unterschiedliche Familienformen repräsentieren sollen. Die Teilnehmenden sollen sich zunächst die Bilder im Lehrbuch ansehen. Dann initiiert Nancy eine für den Orientierungskurs typische kulturvergleichende Kommunikation, die im Unterschied zur vorherigen, von Hasib initiierten, von den Teilnehmenden kooperativ vollendet wird.

Nancy: ,Regenbogenfamilien' - Gibt es das in Russland?

Tatjana: Nein, nein.

Nancy: Wie ist es in Iran?

Hasib: Nein, nein.

Nancy: Wie ist es in Eritrea oder im Sudan?

Amjad: Nicht, auch nicht im Sudan.

Die Grundstruktur des von der Lehrkraft initiierten spontanen Kulturvergleichs im Unterrichtsgespräch folgt einer wiederkehrenden Dramaturgie. Die Lehrkraft greift ein kulturelles Phänomen oder einen normativen Wert aus dem behandelten Themenbereich heraus und fordert die Teilnehmenden dazu auf, diesen auf ihr Herkunftsland zu beziehen. Der Ablauf dieser Sequenzen weist, wie das übrige Unterrichtsgespräch auch, große Ähnlichkeiten zur Didaktik des fragend-entwickelnden Unterrichts auf. Die Lehrkraft hat einen narrativen Plot, den die Teilnehmenden nicht kennen. Sie versucht mit gezielten Fragen das Gespräch in diesen geplanten narrativen Plot zu kanalisieren. Die initiierende Frage beginnt meist mit "Wie ist das in ..." oder wie im vorliegenden Fall mit "Gibt es das in ...". Dann werden entweder die Länder nacheinander genannt, die in der Kurskonstellation vertreten sind oder die Teilnehmenden werden einzeln adressiert und sollen Auskunft darüber geben, wie sich der Sachverhalt in „ihrem Heimatland" verhält. In beiden Fällen werden die Teilnehmenden als Repräsentant*innen und kulturelle 
Expert*innen des Landes angesprochen, dessen Staatsangehörigkeit sie besitzen. Sie werden also nicht nach ihren individuellen Erfahrungen und Einschätzungen gefragt, sondern zu pauschalisierenden Äußerungen über die Gepflogenheiten in ,ihrem' Land und damit der Konstruktion einer homogenen Nationalkultur aufgefordert.

Im vorliegenden Fall greift Nancy mit der ,Regenbogenfamilie' zunächst eine Lebensform heraus, die in Deutschland seit einiger Zeit rechtlich möglich ist, jedoch quantitativ im Vergleich mit heterosexueller Elternschaft eher selten vorkommt. Auch wird gleichgeschlechtliche Elternschaft in Deutschland durchaus unterschiedlich bewertet, kann also kaum als uneingeschränkt gesellschaftlich akzeptiert gelten. Gleichzeitig sind mit der Bezeichnung, Regenbogenfamilie' und den diese Familienform legitimierenden Rechtsnormen bereits bedeutsame Symbole gesellschaftlicher Akzeptanz verbunden. Nancy greift mit anderen Worten aus der Vielzahl von in Deutschland legalen Familienformen eine heraus, deren moralische Legitimität umstritten ist und die quantitativ nicht den Status einer typischen Familienform hat. Sie bekräftigt damit allerdings den Deutungsrahmen, den die Übung im Lehrbuch offeriert, welche eine Klassifikation und visuelle Repräsentanz der Diversität an möglichen Familienformen unter der Überschrift "Wie leben die meisten Menschen in Deutschland zusammen?" (eigene Hervorhebung) herstellt. Demnach gibt es neben der traditionellen auch modernisierte Familien, "Neue Eltern" und Gemeinschaften sowie Kinderlose. ${ }^{51}$

Betrachten wir die Struktur des Vergleichs nun etwas genauer. Wie ist ihr interaktiver Verlauf, welche Einheiten werden unter der Annahme der Vergleichbarkeit und Differenz miteinander relationiert und was wird als Tertium Comparationis eingeführt? Zunächst fällt auf, dass Nancy mit "Gibt es das in ..." eine geschlossene Frageform wählt, die einen differenzierten Vergleich zwischen Familienformen in verschiedenen Ländern ausschließt. Die gesamte Sequenz enthält drei „Gibt es ..."--Fragen, deren Dramaturgie Auskunft über ihren narrativen Plot geben. Die erste bezieht sich auf die Existenz einer bestimmten Lebensform, die zweite und dritte auf die morali-

$51 \mathrm{Vgl}$. Butler/Würtz/Kotas et al. (2017) 100 Stunden Deutschland, S. 19. sche Bewertung bzw. Bestrafung von Frauen, die gegen Traditionen verstoßen.

Statt die Vielfalt an Lebensformen in Deutschland mit jener in den Herkunftsländern der Teilnehmenden oder der den Teilnehmenden persönlich bekannten Lebensformen zu vergleichen, sollen die Befragten zunächst Auskunft darüber geben, ob es in dem jeweiligen Land die Familienform ,Regenbogenfamilie' gibt. Auffällig ist hier, dass Nancy nicht fragt, ob es gleichgeschlechtliche Paare gibt, die gemeinsam Verantwortung für ein Kind übernehmen, sondern ganz explizit den ausschließlich in Deutschland bekannten Begriff ,Regenbogenfamilie' verwendet. Diese Frage können und müssen die Teilnehmenden einhellig mit einem eindeutigen und teilweise durch Wortwiederholung bekräftigten ,Nein' beantworten. Es ist zu vermuten, dass diese Antwort von Nancy erwartet wurde, zumindest bedarf sie offensichtlich keiner weiteren Erläuterung. Der Kommentar der Teilnehmerin Tatjana, die mit der Bemerkung, dass Frauen immer der Kopf der Familie seien (sie bezieht sich hier vermutlich auf russische Familien) einen anderen Aspekt zum Thema Familienformen einbringen möchte, wird nicht aufgegriffen. Stattdessen will Nancy daraufhin von Tatjana wissen, ob positiv oder negativ über Frauen gesprochen wird, die zum zweiten Mal heiraten, was Tatjana mit "negativ" beantwortet. Daran schließt Nancys dritte Frage, die sich diesmal an Hasib richtet:

\footnotetext{
Nancy: Dürfen Menschen ein Kind bekommen, ohne verheiratet zu sein?

Hasib: Ich verstehe nicht.

Nancy: Gibt es ein Gesetz, welches - danach erlaubt ist, Kinder ohne Ehe zu haben?

Hasib: Nein, aber manchmal bekommt eine Frau ein Kind ohne Heirat. Danach heiraten.

Nancy: Muss sie ins Gefängnis oder muss sie sterben? Wenn sie ein Kind hat.

Hasib: Sie hat dann Probleme [Pause] das weiß ich nicht.
}

Auch diese Fragesequenz folgt dem gleichen Muster. Sie beginnt mit einer geschlossenen Frage, die mit "Ja" oder "Nein" zu beantworten ist und sie geht von einer in Deutschland existierenden Norm aus, die daraufhin befragt wird, ob sie in Iran auch vorhanden ist. Interessant ist nun der weitere Verlauf der Gesprächssequenz. Anders als Tatjana zeigt Hasib nicht das von inm erwartete 
Antwortverhalten. Zunächst weist er die Frage als "nicht verstanden" zurück, so wie es Nancy zuvor mit seiner Frage getan hatte. Anders als Hasib zuvor gibt aber Nancy an dieser Stelle nicht auf, sondern paraphrasiert ihre Frage in einer weiteren "Gibt es ..."-Frage. Hasib kommt nun der Aufforderung nach und antwortet gemäß der geschlossenen Frage (und vermutlich auch der Erwartung der Lehrkraft) mit "Nein", schränkt dieses "Nein" jedoch ein, indem er ein "Aber" hinzufügt. Demnach kommt es durchaus vor, dass unverheiratete Frauen Kinder bekommen, sie heiraten dann aber nach der Geburt. Diese Antwort scheint für Nancy nicht befriedigend zu sein, weshalb sie eine weitere Frage anschließt, die diesmal die Form einer Alternativfrage hat. Sie bietet Hasib zwei Antwortmöglichkeiten, zwischen denen er wählen kann, beide Antworten unterstellen eine grundsätzliche Strafbarkeit und massive Strafverfolgung nichtehelicher Mutterschaft in Iran: Freiheitsberaubung oder Tod. Hasib kann diese Frage im Horizont der angebotenen Antwortmöglichkeiten nicht beantworten. Er bietet eine dritte Alternative an, welche die Vermutung der Strafbarkeit und Strafverfolgung nicht bestätigt, wohl aber nichteheliche Mutterschaft als für die Betroffene problematische Lebenssituation bezeichnet.

Hasib bringt in dieser Gesprächssequenz ein Normalitätsverständnis zum Ausdruck, das sich von jenem unterscheidet, welches Nancy als in Deutschland realisiertes, jedoch im Prinzip universell gültiges annimmt. Hasib sagt, das kommt schon vor, dass Frauen in Iran ein Kind bekommen, ohne verheiratet zu sein, aber es steht für ihn ganz außer Frage, dass die Frau, wenn sie ein Kind hat, heiraten wird. Es ist also gar nicht vorgesehen und erscheint inm auch einigermaßen unvorstellbar, dass man als Frau in Iran auf die Heirat verzichten würde, wenn man ein Kind bekommen hat. Hasib spricht also von einer gesellschaftlichen Konvention, von einer als kollektiv geteilt angenommenen moralischen Auffassung darüber, dass es richtig ist, verheiratet zu sein, wenn man Kinder hat und falsch, wenn man unverheiratet Kinder hat oder zusammenlebt (wie er zu einem früheren Zeitpunkt zu verstehen gab). Nancy hingegen geht von einer als kollektiv geteilt angenommenen moralischen Auffassung aus, dass individuelle Freiheitsrechte über allem anderen stehen, aus welchen sich ableiten lässt, dass Frauen sich individuell für ein Kind und gegen eine Heirat aussprechen können. Sie nimmt weiter an, dass diese individuellen Freiheitsbestrebungen in allen Menschen, auch in Iran vorhanden, dort aber staatlich unterdrückt und gesetzlich sanktioniert werden.

Befragt auf das Tertium Comparationis und die von den Beteiligten angenommenen Vergleichseinheiten lässt sich an dieser Gesprächssequenz zwischen Nancy und Hasib Folgendes feststellen. Die Vergleichseinheiten Deutschland und Iran werden von Nancy eingeführt und von Hasib widerspruchslos angenommen. Was aber ist das Tertium Comparationis in diesem Fall und wie wird es gebildet? Mit der Frage nach der rechtlichen Möglichkeit zur nichtehelichen Mutterschaft in Iran und der Art der daran anschließenden Folgefragen nach dem Strafmaß macht Nancy deutlich, dass sie davon ausgeht, in Iran stehe nichteheliche Mutterschaft unter schwerer Strafe. Sie stellt damit einen Kontrast zur gesellschaftlichen Situation in Deutschland her. Den Teilnehmenden waren ja zuvor durch die Darstellung im Lehrbuch nichteheliche, ebenso wie alleinerziehende Elternschaft als mögliche und gesellschaftlich etablierte Lebensformen vorgestellt worden. In diesem kontrastierenden Vergleich wird nun durch die spezifische Dramaturgie der Fragen das Vergleichskriterium ,Lebensformen' aus dem Lehrbuch im Unterrichtsgespräch in ,Frauenrechte' transformiert. Bereits die Frage an Tatjana fokussierte auf die Situation von Frauen in deren Herkunftsland. Auf diese Weise stützt Nancy ihren mittels fragend-entwickelnder Gesprächsführung entfalteten narrativen Plot, den man als ,Modernisierungsnarrativ' bezeichnen und wie folgt zusammenfassen kann: In Deutschland herrschte früher ein traditionelles Familienmodell vor, das die persönlichen Freiheits- und Entfaltungsrechte stark einschränkte, was insbesondere zu Lasten der Frauen ging. Dieses hat sich aber inzwischen gewandelt, so dass Deutschland zu einem Land geworden ist, in dem individuelle Freiheitsrechte, Gleichberechtigung und Toleranz gegenüber anderen Lebensformen sich als moralische Grundwerte etabliert haben. Der Kulturvergleich dient hier dazu, die Fortschrittlichkeit und Attraktivität der für Deutschland als maßgeblich angenommenen moralischen Grundwerte und Lebensweisen zu unterstreichen. Die Teilnehmenden werden dann durch spezifische Fragen ange- 
leitet, Defizite in ihrem Herkunftsland festzustellen und die als eigen zugeschriebene bzw. reklamierte Herkunftskultur gegenüber der deutschen Zielkultur als minderwertig zu, erkennen'. Das Tertium Comparationis ist hierbei allerdings kein Drittes, vor dem die Lebensweisen hier und dort vergleichbar würden, sondern es werden Begriffe universalisiert, die an der gesellschaftlichen Erfahrung der Zielkultur gebildet wurden und diese zum Vergleichsmaßstab erklären. Es handelt sich hierbei nicht um einen Vergleich im eigentlichen Sinne, sondern um ein Wechselspiel zwischen "Nostrifizierung", der "Aneignung des Anderen nach eigenem $\mathrm{Maß}$ "52 und "Othering", der Konstruktion des ,Anderen' als Prozess des "Different-Machens." 53 Damit wird unterstrichen, "dass die Markierung der ,Anderen' mit der Etablierung der dominanten Position der ,Normalen' einhergeht." 54 Um diese Unterschiede und Gegenüberstellungen zwischen ,Wir' und ,Ihr' zu erklären, werden die Kursteilnehmenden ,geandert', es wird innen mithin "kulturelle Andersheit und/oder defizitäre Eigenschaften individuell wie kollektiv zugeschrieben". ${ }^{55}$

\subsection{Zusammenfassung: Funktionen und invektive Qualitäten des Kulturvergleichs}

Wir haben in diesem Beitrag den Kulturvergleich im Orientierungskurs als kommunikative Gattung auf seine außen- und binnenstrukturellen Merkmale und Variationen beschrieben und seine situative Realisierung exemplarisch in Augenschein genommen, um den Vergleichsvorgang und die jeweils eingeführten Tertia Comparationis zu bestimmen. Dabei konnten Einblicke in das kulturelle Wissen gewonnen werden, welches sich im Kulturvergleich als kommunikativer Gattung sedimentiert. Ebenfalls konnte nachvollzogen werden, wie sich dieses Wissen im Unterrichtsgespräch aktualisiert und mit sozialstrukturellem Ordnungswissen verknüpft. Während sich die narrativen Plots von Lehrkraft zu Lehrkraft unterscheiden mögen, so lässt sich doch eine

52 Matthes (1992) Operation "Vergleichen", S. 84. 53 Castro Varela/Dhawan (2015) Postkoloniale Theorie, S. 164 .

54 Ivanova (2016) Umgang der Migrationsanderen, S. 42. 55 Kunz (2016) Ungleichheit, S. 251. übereinstimmende Grundstruktur ausmachen. Wir können damit mit relativ großer Sicherheit sagen, dass es sich bei der am exemplarischen Fall rekonstruierten Herstellung einer asymmetrischen Vergleichskonstellation zwischen abgewerteter Herkunfts- und aufgewerteter Zielkultur um ein ordnungskonstitutives Muster handelt, dass den Kulturvergleich im Orientierungskurs entgegen seiner erklärten didaktischen Zielsetzung zu einer invektiven Gattung macht. Seine konstitutive Bedeutung wird in der Häufigkeit seines Vorkommens und in der wiedererkennbaren Musterhaftigkeit seines Ablaufs deutlich. Darüber hinaus findet sie sich in metainvektiven Äußerungen von Teilnehmenden bestätigt. ${ }^{56}$

Der Kulturvergleich im Orientierungskurs kann folglich als invektive Gattung begriffen werden, insofern Kulturen primär als territorial in Staatswesen verfasste Nationalkollektive definiert werden, welche durch spezifische Vergleichsoperationen einander asymmetrisch zugeordnet werden. Dabei werden primär kulturelle Differenzen und kaum Gemeinsamkeiten thematisiert. Die Zielkultur wird mittels maximal kontrastierender und nostrifizierender Vergleiche als von der Herkunftskultur stark verschieden und ihr gleichzeitig überlegen hergestellt. Die Analyse hat gezeigt, dass die Teilnehmenden in der kulturvergleichenden Kommunikation nicht als Individuen, sondern als "Exemplare"57 der ihnen zugeschriebenen Herkunftskultur angesprochen werden, für die sie gleichzeitig als auskunftspflichtig und -fähig gelten. Nicht ihre durch Sozialisation und im Lebenslauf erworbenen individuellen Erfahrungen stellen

56 Hierzu ein Beispiel aus einem anderen Orientierungskurs: In einer Übung sollten die Kursteilnehmenden eine Situation daraufhin bewerten, ob sie zu den Grundwerten Demokratie und Rechtsstaat passt. In dem Fallbeispiel hatte ein fiktiver Bundespräsident seine Position dazu missbraucht, seinem Neffen eine Stelle zu verschaffen, die dieser ohne die Intervention seines Onkels nicht bekommen hätte. Die Lehrkraft initiierte eine kulturvergleichende Diskussion mit der Frage: „Passiert das in eurem Heimatland?" Reihum bestätigten die Teilnehmenden, dass dies in ihrem Heimatland sicher vorkomme, einer vermutete gar, dies würde für jedes Land gelten. Daraufhin widersprach ein Teilnehmender, in dem er Deutschland von dieser Regel ausnahm („Ja, außer in Deutschland"). Seine Aussage markierte er gestisch als ironischen Kommentar und unterstrich ihren spielerischen Charakter mit Lachen. Er gab damit zu erkennen, dass er die invektiven Konstitutionsmerkmale des Kulturvergleichs im Orientierungskurs durchschaut. 57 Hirschauer/Boll (2017) Un/doing Differences, S. 42. 
die Referenz für den Vergleich dar. Vielmehr werden die Teilnehmenden aufgefordert, national-kulturelle Standards zu konstruieren. Dabei müssen sie auf stereotypes Fremdwissen über ,ihr' Land bzw. ,ihre' Kultur bezugnehmen, welches in der kulturvergleichenden Operation von Lehrkräften und anderen Teilnehmenden herangezogen wird.

Wir haben schließlich festgestellt, dass diese Praxis vom fremdsprachendidaktischen Konzept des wertfreien Kulturvergleichs abweicht. Damit ist jedoch das Ziel dieses Beitrags noch nicht erreicht. Unser primäres Anliegen besteht nicht darin, die gängige Praxis zu kritisieren oder zu problematisieren. Vielmehr wollen wir nun abschließend eine These zu der Frage formulieren, welche Probleme die kommunikative Gattung des Kulturvergleichs im Orientierungskurs löst. Diese Frage führt zurück zu den eingangs identifizierten Spannungsmomenten zwischen den didaktischen Leitlinien denen der Orientierungskurs folgt und seiner integrationspolitischen Funktion. Wir nehmen an, dass mit der Herstellung von asymmetrischen Kontrasten zwischen Herkunfts- und Zielkultur die Lehrkräfte das Problem lösen, das sich aus dem Verbot der Indoktrination einerseits und dem Auftrag zur Wertebildung andererseits ergibt. Die Teilnehmenden dürfen also in den Kursen nicht explizit dazu aufgefordert werden, ein positives Verhältnis zum deutschen Staat zu entwickeln, sie werden stattdessen dazu gebracht, die Wissenstraditionen und Werteorientierungen, die sie im bisherigen Lebenslauf erworben haben, durch die nostrifizierenden Augen der Zielkultur neu zu bewerten, wobei der Vergleich so angelegt ist, dass erstens die individuelle Erfahrung durch Stereotypen ersetzt wird und zweitens die Bewertung immer zugunsten der Zielkultur ausfällt.

Die Ergebnisse unserer bisherigen Analyse zeigen, dass der Kulturvergleich als invektive Gattung eine wichtige Funktion im Orientierungskurs hat. Dabei zeigen sich in den Kurskonstellationen durchaus Unterschiede, die in der weiteren Analyse näher in Augenschein genommen werden müssen. Welche Möglichkeiten des Widerspruchs oder des Widerstands haben etwa die Kursteilnehmenden? Welche unterschiedlichen narrativen Plots verfolgen die Lehrkräfte und inwieweit spiegeln diese die Vielfalt an Wissenstraditionen und moralischen Orientierungen innerhalb Deutschlands wider? Welche Bedeutung hat dabei das korrektive Feedback, das als typisches kom- munikatives Muster des Fremdsprachenunterrichts auch im Orientierungskurs prominent ist, hier jedoch in Verbindung mit der Vermittlung von kulturellem Wissen auftritt? Für die Beantwortung der Frage, mit welchen institutionellen und kommunikativen Mitteln die Tradierung und Kanonisierung von Wissenstraditionen unter der Bedingung von sozialer Heterogenität und kultureller Diversität organisiert wird, ist der Orientierungskurs in jedem Fall ein aufschlussreiches Forschungsfeld.

\section{Literaturverzeichnis}

\section{Quellen}

BAMF (2017, April): Curriculum für einen bundesweiten Orientierungskurs. URL: https://www.bamf.de/ SharedDocs/Anlagen/DE/Integration/Integrationskurse/Kurstraeger/KonzepteLeitfaeden/ curriculum-orientierungskurs-pdf.pdf?_blob=publicationFile (letzter Zugriff: 31.12.20).

Butler, Ellen/Würtz, Helga W./Kotas, Ondřej et al. (2017): 100 Stunden Deutschland - Orientierungskurs Politik, Geschichte, Kultur. Kurs- und Übungsbuch mit Audios online. Stuttgart: Klett.

Schote, Joachim (2017): Orientierungskurs. Grundwissen Politik, Geschichte und Gesellschaft in Deutschland. Berlin: Cornelsen.

\section{Forschungsliteratur}

Abu-Lughod, Lila (1991): Writing Against Culture. In: Fox, Richard (Hg.): Recapturing Anthropology. Working in the Present. Santa Fe: School of American Research Press, S. 137-162.

Berg, Eberhard/Fuchs, Martin (1993): Kultur, soziale Praxis, Text. Die Krise der ethnographischen Repräsentation. Frankfurt a.M.: Suhrkamp.

Bergmann, Jörg R./Luckmann, Thomas (1995): Reconstructive Genres of Everyday Communication. In: Quasthoff, Uta M (Hg.): Aspects of Oral Communication. Berlin: De Gruyter, S. 289-304.

Betz, Emma (2017): Diskursmarker aus konversationsanalytischer Sicht: Prosodisch integriertes ja am Beginn von responsiven Turns. In: Hardarik Blühdorn, Hadarik/Arnulf Deppermann, Arnulf/Helmer, Henrike/ Spranz-Fogasy, Thomas (Hgg.): Diskursmarker im Deutschen Reflexionen und Analysen. Göttingen: Verlag für Gesprächsforschung, S. 183-205.

Bollig, Michael (2003): Interkulturelle Vergleichsverfahren. In: Beer, Bettina/Fischer Hans (Hgg.): Ethnologie. Einführung und Überblick. Berlin: Reimer, S. 391-412.

Castro Varela, María do Mar./Dhawan, Nikita (2015): Postkoloniale Theorie. Eine kritische Einführung. 2., 
vollständig überarbeitete und erweiterte Auflage. Bielefeld: transcript.

Chen, Eva V. (2013): Das Eigene und das Fremde? In: Magali, Moura/Chen, Eva V. (Hgg.): Kulturdidaktik im Unterricht Deutsch als Fremdsprache: Facetten der Vermittlung von Kultur und Landeskunde. Rio de Janeiro: APA Rio.

Clifford, James/Marcus, George E. (1986): Writing Culture: The Poetics and Politics of Ethnography Berkeley/Los Angeles/London: University of California Press.

Dirim, İnci/Wegner, Anke (2018): Normative Grundlagen und reflexive Verortungen im Feld DaF_DaZ*. Leverkusen-Opladen/Berlin: Budrich.

Ellerbrock, Dagmar/Koch, Lars/Müller-Mall, Sabine/ Münkler, Marina/Scharloth, Joachim/Schrage, Dominik/Schwerhoff, Gerd (2017): Invektivität Perspektiven eines neuen Forschungsprogramms in den Kultur und Sozialwissenschaften. In: Kulturwissenschaftliche Zeitschrift 2/1, S. 2-24.

Fornoff, Roger (2018): Migration, Demokratie, Werte: Politisch-kulturelle Bildung im Kontext von Deutsch als Zweitsprache. Göttingen: Universitätsverlag.

Gehring, Wolfgang (2018): Fremdsprache Deutsch unterrichten: Kompetenzorientierte Methoden für DaF und DaZ. Oldenburg: UTB.

Günthner, Susanne/Knoblauch, Hubert (1994): „Forms are the food of faith": Gattungen als Muster kommunikativen Handelns. In: Kölner Zeitschrift für Soziologie und Sozialpsychologie 46/4, S. 693-723.

Haase, Peter/Höller, Michaela (2017): Kulturelles Lernen im DaF/DaZ-Unterricht. Göttingen: Universitätsverlag.

Hirschauer, Stefan/Boll, Tobias (2017): Humandifferenzierung. Modi und Grade sozialer Zugehörigkeit In: Hirschauer, Stefan/Boll Tobias (Hg.): Un/doing Differences. Praktiken der Humandifferenzierung. Weilerswist: Velbrück Wissenschaft, S. 29-54.

Hu, Adelheid: Art. ,Kulturvergleich'. In: Barkowski, Hans/ Krumm, Hans-Jürgen (Hgg.): Fachlexikon Deutsch als Fremd- und Zweitsprache. Tübingen: Francke 2010, S. 178.

Ivanova, Michela (2017): Umgang der Migrationsanderen mit rassistischen Zugehörigkeitsordnungen. Strategien, Wirkungsweisen und Implikationen für die Bildungsarbeit. Bad Heilbrunn: Klinkhardt.

Knoblauch, Hubert/Luckmann, Thomas (2003): Gattungsanalyse. In: Flick, Uwe/Kardoff, Ernst von/Steinke, Ines (Hgg.): Qualitative Forschung. Ein Handbuch. Reinbek bei Hamburg: Rowohlt, S. 538-546.

Kunz, Thomas (2016): Ungleichheit. In: Mecheril, Paul (Hg.): Handbuch Migrationspädagogik. Weinheim, Basel: Beltz, S. 243-260.

Luckmann, Thomas (1986): Grundformen der gesellschaftlichen Vermittlung des Wissens: Kommunikative Gattungen. In: Kölner Zeitschrift für Soziologie und Sozialpsychologie. Sonderheft 27, S. 191-211.

Luckmann, Thomas (1992): Einleitung zu 'Rekonstruktive Gattungen'. Konstanz, Fachgruppe Soziologie: Manuskript.
Matthes, Joachim (1992): The Operation Called "Vergleichen". In: Matthes, Joachim (Hg.): Zwischen den Kulturen? Die Sozialwissenschaften vor dem Problem des Kulturvergleichs. Göttingen: Schwartz, S. 75-99.

Mecheril, Paul (2003): Prekäre Verhältnisse. Über natio-ethno-kulturelle (Mehrfach-) Zugehörigkeit. Münster: Waxmann.

Ott, Josephine (2020): Arbeitspapier des SFB 1285: ,Invektivität und Interkulturalität'. Konzeptionelle Überlegungen und eine annotierte Literaturauswahl. Dresden. URL: https://tud.qucosa.de/landing-page/ https $\% 3 \mathrm{~A} \% 2 \mathrm{~F} \% 2 \mathrm{Ftud}$.qucosa.de $\% 2 \mathrm{Fapi} \% 2 \mathrm{Fquco}-$ sa\%253A72010\%2Fmets\%2F/(22.10.2021) (letzter Zugriff 31.12.20).

Thomas, Alexander (Hg.) (1993): Kulturvergleichende Psychologie. Eine Einführung. Göttingen: Hogrefe.

Weimann, Gunther/Hösch, Wolfram (1993): Kulturverstehen im Deutschunterricht. Ein Projekt zur Lehrerfortbildung. Informationen Deutsch als Fremdsprache 20/5, S. 514-523.

Zeuner, Ulrich (2017): Landeskunde und interkulturelles Lernen. Eine Einführung. Dresden. URL: https:// tu-dresden.de/gsw/slk/germanistik/daf/ressourcen/ dateien/dateien/materialien_zeuner/zeuner_reader_ landeskunde.pdf?lang=de (letzter Zugriff: 22.10.2021). 